\title{
QUAL O MELHOR HORÁRIO DA TOMADA DA MEDICAÇÃO ANTI-HIPERTENSIVA?
}

\author{
WHAT IS THE BEST TIME TO TAKE ANTIHYPERTENSIVE MEDICATION?
}

Juliana Tranjan Coragem', Thiago de Souza Veiga Jardim²

\section{RESUMO}

A hipertensão arterial é a principal causa de morte precoce no mundo. Existem diversos fatores que contribuem para sua variação dentro da fisiologia do ritmo circadiano, e que exercem forte impacto sobre o controle da pressão arterial (PA). Os valores da PA sofrem interferência de acordo com o horário da medida. Nas primeiras horas da manhã PAS aumenta rapidamente em 20 a 25 mmHg e a PAD em 10 a 15 mmHg. As drogas anti-hipertensivas, sofrem diversas influências na sua absorção, metabolização e excreção dependentes do ritmo circadiano e de suas propriedades físico-químicas. O ciclo circadiano exerce grande impacto no controle da PA, e apenas modificando o horário das prescrições, seguindo o ritmo circadiano, talvez seja possível obter melhor controle pressórico com menor dose da medicação, reduzindo assim, as possíveis reações adversas.

Descritores: Hipertensão; Cronoterapia; Pressão Arterial; Ritmo Circadiano; Doença Cardiovascular.

\section{ABSTRACT}

Hypertension is the leading cause of early death worldwide. Several factors contribute to its variation within the physiology of circadian rhythm, and these factors have a strong impact on blood pressure (BP) control. BP values are influenced by the measurement time. In the early hours of the morning, SBP increases rapidly by 20 to $25 \mathrm{mmHg}$ and DBP by 10 to $15 \mathrm{mmHg}$. Antihypertensive drugs suffer from a variety of influences on their absorption, metabolism and excretion, depending on the circadian rhythm and its physical-chemical properties. The circadian cycle has a great impact on BP control, and only by changing the schedule of prescriptions, following the circadian rhythm, it may be possible to obtain better pressure control with a lower dose of medication, thus reducing potential adverse reactions.

Keywords: Hypertension; Chronotherapy; Arterial Blood Pressure; Circadian Rhythm; Cardiovascular Diseases.

\section{INTRODUÇÃO}

Sabe-se que a hipertensão arterial é considerada a principal causa de morte precoce, levando a aproximadamente 10 milhões de mortes em todo o mundo. ${ }^{1}$ No Brasil, os dados do VIGITEL (2019) demostram que a prevalência de hipertensão aumentou, acometendo $59,3 \%$ dos adultos com 65 anos ou mais (55,5\% dos homens e $61,6 \%$ das mulheres) e mais de $20 \%$ da população adulta (maiores de18 anos). ${ }^{2,3}$ Devido à grande prevalência da hipertensão arterial e suas consequências é imprescindível que seu tratamento seja feito de forma adequada para prevenir complicações.

No mundo todo os médicos mantem a prática de orientar seus pacientes a ingerirem suas medicações anti-hipertensivas pela manhã, mas este seria realmente o melhor horário para a tomada desta medicação? Seria essa a melhor opção para se atingir o alvo pressórico com maior eficácia?

Ao prescrever medicações, sejam elas anti-hipertensivas ou qualquer outra classe de fármaco é importante se lembrar do ciclo circadiano, para que ela possa ter o melhor efeito com a menor dose efetiva, reduzindo assim, as possíveis reações adversas. ${ }^{4}$
Cada célula do organismo possui seu ritmo de funcionamento próprio. Os padrões diários de comportamento como o ciclo sono e vigília, horas de sono, horário das refeições, tempo de jejum, interferem diretamente com a fisiologia do organismo. ${ }^{4}$ Além disso, é importante ressaltar que existem também fatores inerentes à droga como sua farmacocinética e suas propriedades físico-químicas. Tais fatores podem sofrer interferências na absorção de acordo com o horário da tomada da medicação e a associação com alimentação. Por esses motivos é necessário conhecer o ciclo circadiano e as características das drogas que serão prescritas.

Os tratamentos para hipertensão arterial, atualmente empregados são: os tratamentos não farmacológicos como mudanças de estilo de vida, e a terapia farmacológica. Caso não se atinja o alvo pressórico, a tendência é que se aumente a dose da medicação ou que se associe mais uma droga. Porém, alguns estudos avaliaram o impacto do ciclo circadiano no controle da pressão arterial, apenas modificando o horário do uso das medicações, sem aumentar o número de drogas prescritas. ${ }^{5}$

1. Programa de Pós-Graduação em Ciências da Saúde - Universidade Federal de Goiás, Goiania, GO, Brasil.

2. Hospital do Coração de Goiás, Goiânia, Goiás, Brasil.

Correspondência: Thiago de Souza Veiga Jardim. R. 235, s/n - Setor Leste Universitário, Goiânia - G0, 74605-050. thiagoloirin@hotmail.com

http://dx.doi.org/10.47870/1519-7522/20202704138-40 


\section{CICLO CIRCADIANO}

O ritmo biológico é visto como alterações adaptativas dos nossos ancestrais devido a mudanças ambientais ocorridas nas 24 horas do dia, nos meses e nos anos. Os seres humanos herdaram mecanismos de funcionamento do relógio biológico únicos de seus antepassados. ${ }^{6}$ Nos mamíferos, o marcapasso biológico dominante está na parte anterior do hipotálamo no núcleo supraquiasmático (SCN). O período biológico herdado do ciclo circadiano humano não é exatamente de $24 \mathrm{~h}$, em algumas pessoas pode ser mais longo ou mais curto em outras, os mediadores que atuam no SCN sofrem diversas interferências, como o claro-escuro, além disso, outros fatores ambientais trazem o ciclo para $24 \mathrm{~h} .{ }^{6,7}$

A rotina do sono, entre outros fatores, interfere no pico do ritmo circadiano levando em consideração um período de $24 \mathrm{~h}$. O pico da secreção ácida gástrica, da contagem das células brancas, da secreção dos peptídeos natriuréticos atriais e da proteína relacionada ao gene da calcitonina ocorrem tarde da noite ou no início do sono. Já a secreção do hormônio do crescimento $(\mathrm{GH})$, do hormônio estimulador da tireoide (TSH), o número de linfócitos e eosinófilos, a concentração plasmática de melatonina e calcitonina ocorrem mais tarde no sono, assim como o hormônio adrenocorticotrófico (ACTH), o hormônio folículo estimulante (FSH) e o hormônio Luteinizante (LH). A quantidade de colesterol e triglicérides sérico são maiores no início da noite. Já a secreção de cortisol, a atividade da renina, angiotensina e aldosterona são maiores pela manhã, assim como a complacência arterial, resistência vascular periférica e agregação plaquetária, isto pode explicar em parte, a maior incidência de infarto agudo do miocárdio, morte súbita e ataque isquêmico transitório no período da manhã, nas primeiras horas após despertar.,

As variações da pressão arterial correlacionam-se ao ciclo circadiano e dependem de uma série de fatores como a etnia, sexo, tônus do sistema nervoso autônomo, hormônios vasoativos e também as variações da função renal. Além disso, a pressão arterial também sofre influência de fatores externos como a temperatura e humidade do ambiente, fatores emocionais, consumo de cafeína, álcool e a rotina de sono. ${ }^{9}$

A medida da pressão arterial (PA) sofre interferência de acordo com o horário da medida. A pressão arterial sistólica (PAS) e diastólica (PAD) apresentam uma variação considerável ao longo do dia. Nas primeiras horas da manhã PAS aumenta rapidamente em 20 a $25 \mathrm{mmHg}$ e a PAD em 10 a $15 \mathrm{mmHg}$. Entretanto elas são maiores no período da tarde, no início da noite a tendência é que a PA decline, atingindo níveis mais baixos no sono. ${ }^{6,10}$ Estudos demonstram que o aumento da PA matinal coincide com o início da atividade diurna e se torna um fator de risco independente de acidente vascular cerebral (AVC). Além disso, a ausência de declínio da PA em 10 a $20 \%$ durante o sono, está associado a um aumento no risco de lesão de órgão alvo. ${ }^{11,12}$ Diversos estudos demostraram que a pressão arterial durante o sono é um melhor preditor de risco para eventos cardiovasculares e lesão de órgão alvo em relação à medida da PA diurna ou a média das 24 h. ${ }^{12-17}$

As fases do relógio circadiano determinam quando os sintomas das doenças tendem a se intensificar, podendo afetar as respostas aos testes diagnósticos e às intervenções medicamentosas. ${ }^{6}$ Sendo assim, é imprescindível conhecer as alterações do ritmo circadiano e a história natural das doenças, para que o tratamento seja instituído no melhor horário.

\section{CRONOTERAPIA DOS ANTI-HIPERTENSIVOS}

Cronoterapia é definida como o uso de uma medicação, seja ela com tecnologias especiais de liberação ou não, para que suas concentrações séricas e tissulares estejam agindo de acordo com o ritmo circadiano fisiológico, das patologias e dos sintomas, aumentando, desta forma, seu potencial de ação com menores reações adversas. ${ }^{11,12}$

Existem diversos fatores que podem interferir na farmacocinética (FC) da droga tais como: o pH gástrico e o tempo de esvaziamento gástrico, motilidade gastrointestinal, função biliar, taxa de filtração glomerular, atividades das enzimas hepáticas. Entretanto a absorção, distribuição, metabolização e eliminação da droga sofrem variações de acordo com o ritmo circadiano e interferem com a FC da droga. O pico plasmático da droga (Cmax), tempo para atingir a Cmax, a área sobre a curva tempo-concentração (AUC), o volume de distribuição $(V d)$, ligação à proteína, a meia vida da droga e o clearence $(\mathrm{CL})$ são parâmetro farmacocinéticos considerados como constate em tempo, mas na verdade, são ritmo circadiano dependentes. As diferenças na farmacodinâmica de cada droga podem sofrer interferências farmacocinéticas e do ritmo circadiano, alterando a concentração da fração livre da droga, metabolização, clearence e os receptores da droga. Portanto, o ritmo circadiano está sempre presente e influenciando as fases farmacocinéticas. ${ }^{12,16,18}$

Após a ingestão oral do fármaco, suas propriedades físico-químicas, a área e estrutura da biomembrana, tempo de esvaziamento gástrico, fluxo sanguíneo gastrointestinal interferem na sua absorção. Fármacos mais lipolíticos são absorvidos mais rapidamente quando ingeridos pela manhã e a composição dos alimentos podem influenciar nas variações circadianas. ${ }^{18}$

Na prática clínica atual, a maioria das medicações anti-hipertensivas são administradas no período da manhã, contudo, este é o horário em que a sua concentração plasmática está em seu menor nível, é justamente neste momento em que a concentração sérica da medicação deveria ser maior, pois no período da manhã, ao despertar, ocorre elevação rápida dos níveis pressóricos. Através do ponto de vista da cronoterapia, esta medicação deveria ser prescrita no período noturno para reduzir a PA pela manhã. ${ }^{16}$ Entretanto, deve-se levar em consideração que nem todos os pacientes hipertensos apresentam o mesmo padrão de curva pressórica, alguns apresentam um descenso noturno proeminente (dipper), enquanto outros não (não-dipper). Os idosos devem ter uma atenção especial, pelo fato de poderem apresentar uma redução noturna da PA de forma muito acentuada, acarretando efeitos adversos pela hipotensão. ${ }^{16}$

A primeira droga desenvolvida especialmente para a cronoterapia na hipertensão (e angina estável), foi o bloqueador do canal de cálcio (BCC) com início de ação controlada e liberação estendida, o verapamil. Inicialmente foi aprovado nos Estados Unidos da América para uso noturno, para que sua concentração máxima na corrente sanguínea ocorresse pela manhã (entre as 6 e 10H) com uma elevação sustentada durante o dia e declínio de suas concentrações no período noturno. ${ }^{9}$

A maioria dos BBC possuem compostos que promovem liberação lenta da droga, vários artigos investigaram as diferenças entre a administração matutina e noturna dos BCC, entre eles o verapamil, amlodipino, nifedipino, nitrendipino, diltiazem e cilnidipino. ${ }^{11,16,19}$ Lemmer et al. avaliou a farmacocinética de diferentes preparações de nifedipino (liberação rápida x liberação 
lenta, por via oral e endovenosa) em voluntários saudáveis e em hipertensos após a administração da droga em diferentes horários, notou que a biodisponibilidade foi 35\% menor quando administrada a noite em comparação com a dose pela manhã. Os resultados obtidos demostraram que a farmacocinética e os efeitos cardiovasculares dependem do ciclo circadiano. ${ }^{16,19}$

Os inibidores da enzima conversora de angiotensina (IECA) e os bloqueadores dos receptores de angiotensina II (BRA), estão entre as classes anti-hipertensivas mais prescritas em todo o mundo. Elas possuem mecanismos de ação similares.(1) Estudos clínicos realizados com benazepril, enalapril, perindopril, ramipril, lisinopril, demostraram diferenças quando administrados pela manhã e a noite. Em todos os casos, a administração noturna desses medicamentos resultou em um efeito mais marcante na redução da PA noturna e uma modificação significativa do perfil circadiano da PA. O ramipril reduziu de forma mais eficaz a pressão arterial diurna quando administrado pela manhã e reduziu mais efetivamente a PA noturna quando foi administrado à noite..$^{9,20}$

Em um subestudo do consagrado estudo HOPE (Heart Outcomes Prevention Evaluation), sobre monitoramento ambulatorial da pressão arterial (MAPA), as pessoas tratadas com ramipril na hora de dormir apresentaram uma redução acentuada da pressão arterial durante o sono, reduzindo assim, a prevalência de não-dippers. ${ }^{9,11,21}$ Os BRA estão se tornando cada vez mais populares para o tratamento da hipertensão por serem bem tolerados. Um estudo realizado com MAPA durante 48h para avaliar a eficácia anti-hipertensiva da valsartana em pacientes com hipertensão essencial em estágio 1 ou 2 durante 3 meses

\section{REFERÊNCIAS}

1. Williams B, Mancia G, Spiering W, et al. 2018 ESC/ESH Guidelines for the management of arterial hypertension. Eur Heart J. 2018;39(33):3021-104. doi: 10.1093/eurheartj/ehy339. Erratum in: Eur HeartJ. 2019 Feb 1;40(5):475.

2. 2019 VB. Vigitel Brasil 2019. Vigilância de fatores de risco e proteção para doenças crônicas por inquérito telefônico.. Boletim Epidemiológico. 2019 Abril 2020. Acesso em 6/12/2020. Disponível em http://bvsms.saude.gov. $\mathrm{br} / \mathrm{bvs} /$ publicacoes/vigitel brasil 2019 vigilancia fatores risco.pdf

3. Malachias MVB SW, Plavnik FL, Rodrigues CIS, et al. 7a Diretriz Brasileira de Hipertensão Arterial. Arq Bras Cardiol. 2016;107 (3 Suppl 3):1-6.

4. Bowles NP, Thosar SS, Herzig MX, Shea SA. Chronotherapy for Hypertension. Curr Hypertens Rep. 2018;20(11):97. doi: 10.1007/s11906-018-0897-4.

5. Hermida RC, Ayala DE, Fernandez JR, Calvo C. Chronotherapy improves blood pressure control and reverts the nondipper pattern in patients with resistant hypertension. Hypertension. 2008;51(1):69-76. doi: 10.1161/ HYPERTENSIONAHA.107.096933.

6. Smolensky MH, Haus E. Circadian rhythms and clinical medicine with applications to hypertension. Am J Hypertens. 2001;14(9 Pt 2):280S-90S. doi: 10.1016/s0895-7061(01)02175-6.

7. Inouye S-IT, Shibata S. Neurochemical organization of circadian rhythm in the suprachiasmatic nucleus. $1994 ; 20(2)$ :109-30. doi: 10.1016/01680102(94)90029-9.

8. Glasser SP, Frishman W, White WB, Stone P, Johnson MF. Circadian heart rate response to chronotherapy versus conventional therapy in patients with hypertension and myocardial ischemia. Clin Cardiol. 2000;23(7):524-9. doi: $10.1002 /$ clc.4960230711.

9. Hermida RC, Ayala DE, Calvo C, Portaluppi F, Smolensky MH. Chronotherapy of hypertension: administration-time-dependent effects of treatment on the circadian pattern of blood pressure. Adv Drug Deliv Rev. 2007;59(9-10):923-39. doi: 10.1016/j.addr.2006.09.021.

10. Hermida RC, Ayala DE, Mojon A, Fernandez JR. Influence of circadian time of hypertension treatment on cardiovascular risk: results of the MAPEC study. Chronobiol Int. 2010;27(8):1629-51. doi: 10.3109/07420528.2010.510230.

11. Hermida RC, Smolensky MH. Chronotherapy of hypertension. Curr Opin Nephrol Hypertens. 2004;13(5):501-5. doi: 10.1097/00041552200409000-00004.

12. Hermida RC, Ayala DE, Fernandez JR, et al. Administration-time como monoterapia, seja pela manhã ao acordar, a noite ou ao deitar-se. A redução da PA com a dose de $160 \mathrm{mg} /$ dia de valsartana foi semelhante para ambos os horários de tratamento. ${ }^{9,11}$ Como observado com os IECAs, a escolha do horário dos BRAs não apresentou redução das médias de PAS/PAD em vigília, no entanto, a redução das médias de PAS/PAD durante o sono foi mais acentuada em relação ao regime terapêutico matinal, reduzindo a prevalecia de non-dipper. Notou-se ainda, que a ingestão de valsartana, candesartana, e olmesartana, reduziu significativamente a excreção urinária de albumina, quando ingeridas no período noturno, como consequência da redução média da PA durante o sono. ${ }^{20}$

Hermida et al., conduziu um estudo prospectivo, randomizado, para avaliar os efeitos da terapia combinada (valsartan/ amlodipino) em diferentes horários de administração. Os resultados corroboram o efeito significativo de redução da PA em todos grupos de tratamento nos diferentes horários, porem notou-se maior eficácia do controle da PA quando a terapia combinada foi ingerida ao deitar-se, em relação aos

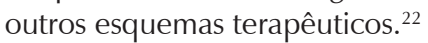

\section{CONCLUSÃO}

Na prática clínica, é importante lembra-se do ritmo circadiano do paciente, da farmacodinâmica e farmacocinética das drogas, desta forma, prescrever um esquema terapêutico individualizado de acordo com as necessidades de cada pessoa, com o objetivo de atingir a meta terapêutica adequada com a menor dose, menor associação medicamentosa e menos reações adversas.

differences in effects of hypertension medications on ambulatory blood pressure regulation. Chronobiol Int. 2013;30(1-2):280-314. doi: 10.3109/07420528.2012.709448.

13. Hermida RC, Crespo JJ, Dominguez-Sardina M, et al. Bedtime hypertension treatment improves cardiovascular risk reduction: the Hygia Chronotherapy Trial. Eur Heart J. 2019 ;ehz754. doi: 10.1093/eurheartj/ehz754.

14. Izzedine H, Launay-Vacher V, Deray G. Abnormal blood pressure circadian rhythm: a target organ damage? Int J Cardiol. 2006;107(3):343-9. doi: 10.1016/j.ijcard.2005.03.046.

15. Noll CA LE, Schmidt A, Coelho EB, Nobre F. Ausência de queda da pressão arterial entre os periodos de vigília e sono. Rev Bras Hipertens 2001; 8(4):468-72.

16. Baraldo $M$. The influence of circadian rhythms on the kinetics of drugs in humans. Expert Opin Drug Metab Toxicol. 2008;4(2):175-92. doi: 10.1517/17425255.4.2.175.

17. Crespo JJ, Dominguez-Sardina M, Otero A, et al. Bedtime hypertension chronotherapy best reduces cardiovascular disease risk as corroborated by the Hygia Chronotherapy Trial. Rebuttal to European Society of Hypertension officials. Chronobiol Int. 2020;37(5):771-80. doi: 10.1080/07420528.2020.1781351.

18. Bruguerolle B. Chronopharmacokinetics. Current status. Clin Pharmacokinet. 1998;35(2):83-94. doi: 10.2165/00003088-199835020-00001.

19. Smith DH. Pharmacology of cardiovascular chronotherapeutic agents. Am J Hypertens. 2001;14(9 Pt 2):296S-301S. doi: 10.1016/s0895-7061(01)02176-8.

20. Hermida RC, Ayala DE, Fernandez JR, Mojon A, Crespo JJ, Rios MT, et al. Bedtime Blood Pressure Chronotherapy Significantly Improves Hypertension Management. Heart Fail Clin. 2017;13(4):759-73. doi: 10.1016/j.hfc.2017.05.010.

21. Heart Outcomes Prevention Evaluation Study Investigators, Yusuf S, Sleight P, Pogue J, Bosch J, Davies R, Dagenais G. Effects of an angiotensinconverting-enzyme inhibitor, ramipril, on cardiovascular events in highrisk patients. N Engl J Med. 2000 Jan 20;342(3):145-53. doi: 10.1056/ NEJM200001203420301. Erratum in: 2000 May 4;342(18):1376. Erratum in: N Engl J Med 2000;342(10):748.

22. Hermida RC, Ayala DE, Fontao MJ, Mojon A, Fernandez JR. Chronotherapy with valsartan/amlodipine fixed combination: improved blood pressure control of essential hypertension with bedtime dosing. Chronobiol Int. 2010;27(6):1287-303. doi: 10.3109/07420528.2010.489167. 